\title{
BMJ Open Contexts and mechanisms that promote access to healthcare for populations experiencing homelessness: a realist review
}

\author{
Rikke Siersbaek (D) , ${ }^{1}$ John Alexander Ford (D) , ${ }^{2}$ Sara Burke (D) , \\ Clíona Ní Cheallaigh (D) , ${ }^{3,4}$ Steve Thomas (iD ${ }^{1}$
}

To cite: Siersbaek R, Ford JA, Burke S, et al. Contexts and mechanisms that promote access to healthcare for populations experiencing homelessness: a realist review. BMJ Open 2021;11:e043091. doi:10.1136/ bmjopen-2020-043091

- Prepublication history and additional material for this paper are available online. To view these files, please visit the journal online (http://dx.doi. org/10.1136/bmjopen-2020043091).

Received 23 July 2020 Revised 17 March 2021 Accepted 19 March 2021

Check for updates

(C) Author(s) (or their employer(s)) 2021. Re-use permitted under CC BY-NC. No commercial re-use. See rights and permissions. Published by BMJ.

${ }^{1}$ Centre for Health Policy and Management, Trinity College Dublin School of Medicine, Dublin, Ireland

${ }^{2}$ Institute of Public Health, University of Cambridge, Cambridge, UK

${ }^{3}$ Clinical Medicine, Trinity College Dublin School of

Medicine, Dublin, Ireland

${ }^{4}$ General Medicine, St James's Hospital, Dublin, Ireland

Correspondence to Ms Rikke Siersbaek; rsiersba@tcd.ie

\section{ABSTRACT}

Objective The objective of this study was to identify and understand the health system contexts and mechanisms that allow for homeless populations to access appropriate healthcare when needed.

Design A realist review.

Data sources Ovid MEDLINE, embase.com, CINAHL, ASSIA and grey literature until April 2019.

Eligibility criteria for selecting studies The purpose of the review was to identify health system patterns which enable access to healthcare for people who experience homelessness. Peer-reviewed articles were identified through a systematic search, grey literature search, citation tracking and expert recommendations. Studies meeting the inclusion criteria were assessed for rigour and relevance and coded to identify data relating to contexts, mechanisms and/or outcomes.

Analysis Inductive and deductive coding was used to generate context-mechanism-outcome configurations, which were refined and then used to build several iterations of the overarching programme theory. Results Systematic searching identified 330 review articles, of which 24 were included. An additional 11 grey literature and primary sources were identified through citation tracking and expert recommendation. Additional purposive searching of grey literature yielded 50 records, of which 12 were included, for a total of 47 included sources. The analysis found that healthcare access for populations experiencing homelessness is improved when services are coordinated and delivered in a way that is organised around the person with a high degree of flexibility and a culture that rejects stigma, generating trusting relationships between patients and staff/practitioners. Health systems should provide long-term, dependable funding for services to ensure sustainability and staff retention.

Conclusions With homelessness on the rise internationally, healthcare systems should focus on highlevel factors such as funding stability, building inclusive cultures and setting goals which encourage and support staff to provide flexible, timely and connected services to improve access.

\section{BACKGROUND}

Homelessness is an extreme expression of social exclusion, experienced by people
Strengths and limitations of this study

This is the first realist review of access to healthcare for homeless populations.

- The review uncovered modifiable contexts and provides important lessons for policy makers working in this area.

- The search strategy was focused on review articles to identify high-level themes, meaning we may have missed relevant sources as well as important information about contextual factors and/or the nature of causative mechanisms in the included literature under review; however, iterative searching was done and an expert panel was engaged in the analysis to offset these potential weaknesses.

lacking a stable and secure place to live. According to the European Typology of Homelessness and Housing Exclusion (ETHOS), homelessness occurs in four ways: rooflessness (sleeping rough, without any shelter); houselessness (having somewhere to sleep but in a temporary shelter or institution); living in insecure housing (eg, insecure tenancies, threat of eviction, violence); and living in inadequate housing (overcrowding, unfit housing, caravans on illegal campsites). ${ }^{1}$ There are various subgroups within the homeless population, including families and single adults. Some single adults have children, but often their children do not live with them. ${ }^{2}$

Populations experiencing homelessness along the full spectrum set out above face both increased difficulty accessing healthcare and poorer outcomes than housed populations, with chronically homeless individuals having worse clinical outcomes than those experiencing intermittent homelessness or as a one-off crisis. ${ }^{3-5}$ Populations experiencing homelessness access primary care less than housed populations and use costly 
unscheduled acute healthcare at a higher rate than their housed peers. $^{5-7}$

Accessing healthcare is complex because it happens along a continuum of need, time and access points, and is delivered in open systems, dependent on many interacting variables on both the supply side (availability, hours, location, staffing, resources, price and so on) and on the demand side (knowledge of health need, availability of the needed time and resources, distance to service location, psychological factors, disposable income and so on). The settings and constructs in which these variables interact are themselves complex (eg, hierarchical, goaldriven cultures in healthcare). As a result, inputs into a health system act in non-linear ways, and at their intersection(s) a variety of intended and unintended outcomes (patients accessing the right care, or inadequate care, or not accessing care all, accessing part of a full course of treatment; patients experiencing stigma or fear or other indirect outcomes; practitioners experiencing stress or professional insecurities; and so on) emerge as more than simply the sum of their parts. ${ }^{8-12}$

In this review we are primarily interested in understanding the health system factors that impact access to healthcare. We conceptualise health system factors and access using the WHO building blocks framework, ${ }^{13}$ including service delivery, health workforce and leadership/governance, and Aday and Andersen's framework for the study of access to medical care, ${ }^{12}$ with health system factors organised under the categories of 'health policy' and 'characteristics of health delivery system'.

Supply-side factors describe the full set of inputs that go into a health system designed to meet the needs of full populations, as much as possible. Meeting the needs of the majority may not adequately meet the needs of groups experiencing social exclusion. In this study we explore how, why, in what circumstances and to what extent these inputs impact healthcare accessibility for the population of interest: single adults experiencing long-term homelessness and complex needs.

Such homeless single adults with complex needs may experience any of the types of homelessness outlined according to ETHOS. We use the term 'populations experiencing homelessness' to refer to this group in this article. The lack of housing in and of itself is not the only driver of poor access to healthcare and poor health outcomes in this population. Homelessness is a marker for and is associated with 'tri-morbidity' - the combination of physical ill health, mental ill health and substance misuse. ${ }^{14}$ In addition to lacking stable housing, populations experiencing homelessness often face a host of health problems and addiction behaviours associated with complex life circumstances and events, including early childhood trauma, having been in care, relationship breakdowns, poverty, lack of employment and more. ${ }^{215}$ While these are common experiences, it is important to stress that they happen to varying degrees and sometimes not at all. ${ }^{15}$

The majority of research exploring access to healthcare for homeless populations has focused on individual-level factors and particularly on why, whether and how individuals experiencing homelessness do or do not access healthcare. ${ }^{36-24}$ A focus on individual-level factors is illuminating, but without understanding and balancing this with health system factors there is a risk that responsibility for accessing services is inappropriately placed with the individual. ${ }^{25} 26$ This review synthesises health system factors that determine healthcare accessibility in order to explore how whole systems can make appropriate healthcare services accessible to populations experiencing homelessness and complex needs.

In this study, we used the WHO's definition of a 'health system':

(i) all the activities whose primary purpose is to promote, restore and/or maintain health; (ii) the people, institutions and resources, arranged together in accordance with established policies, to improve the health of the population they serve, while responding to people's legitimate expectations and protecting them against the cost of ill-health through a variety of activities whose primary intent is to improve health. ${ }^{27}$

For this study we used realist review in the school of Pawson and Tilley ${ }^{28}{ }^{29}$ because it is a methods-neutral approach designed for examining complex interventions or topics by seeking to identify unseen mechanisms which produce a given outcome as well as the contexts in which they are triggered. If activated, mechanisms produce intended and unintended outcomes. Analysis takes place by explaining the causal relationship between contexts, mechanisms and outcomes in the construction of context-mechanism-outcome configurations (CMOCs) (see glossary of terms in online supplemental file 1). We chose to focus on review articles to enable a broader health system lens since an initial scope of the literature showed a substantial body of published literature which would have been unmanageable. Subsequent rounds of searching were undertaken to ensure key literature was not missed.

A review of health system factors which improve access to healthcare for populations experiencing homelessness is needed because an individual-level analysis does not fully explain systemic causes of inadequate access to essential health services. This review sought to answer the following question:

- How, why, for whom, in what circumstances and to what extent can healthcare systems improve access to healthcare for populations experiencing long-term homelessness?

\section{METHODS}

Realist research is a theory-driven approach which seeks to understand causal mechanisms in complex areas of study by asking how, why, for whom, in what circumstances and to what extent something works (or does not work). A realist review approach was chosen because access to healthcare is an open-ended, complex area of study with many entry and exit points for service users and one where 


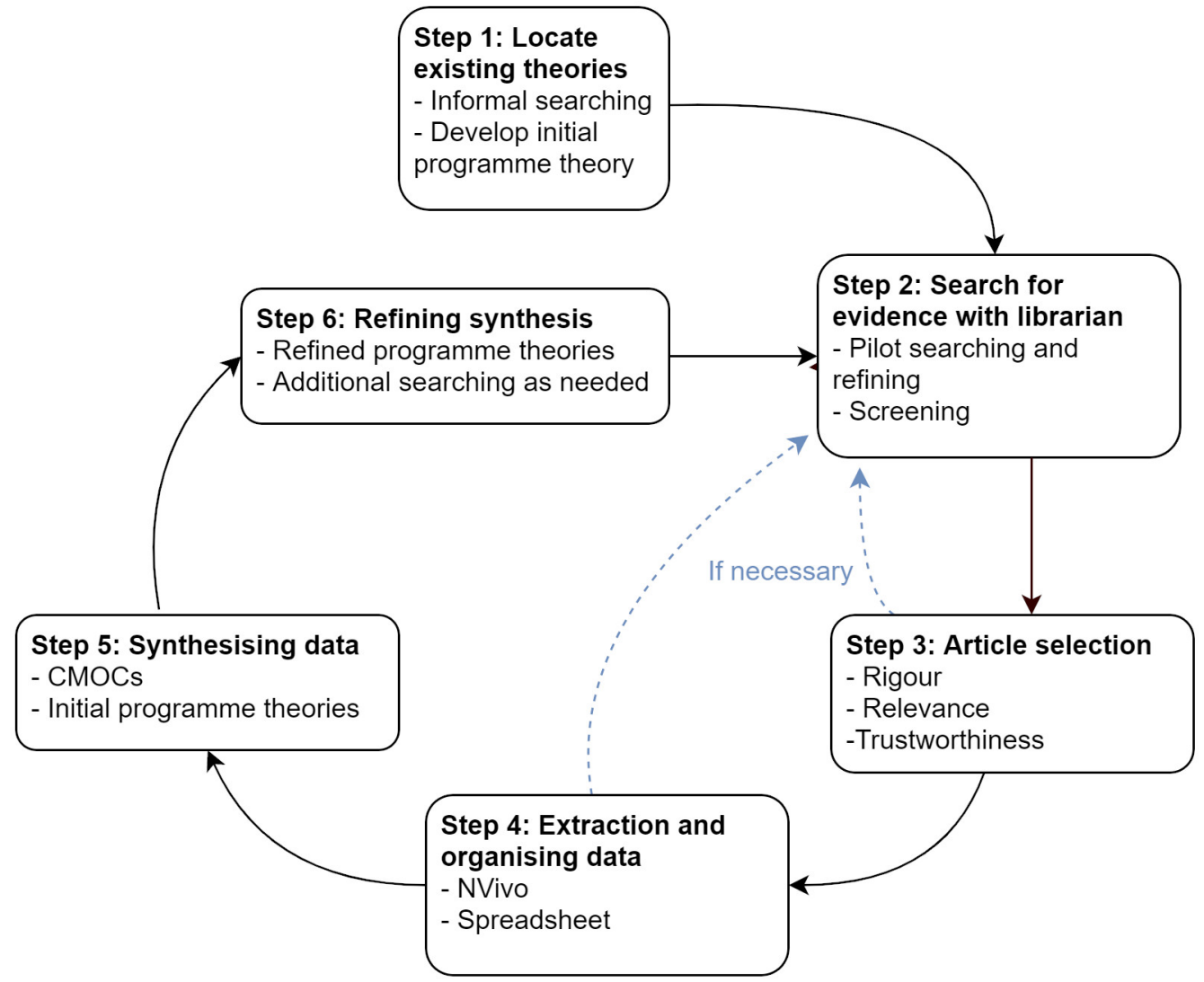

Figure 1 Stages of a realist review. CMOCs, context-mechanism-outcome configuration.

factors interact with each other, creating outcomes (eg, a homeless patient leaving the emergency department (ED) without being seen) that are more than the sum of inputs (eg, ED is nominally accessible because it is always physically open, but the quality of access is impacted by many potential factor, eg, a homeless patient's experiences of stigma). We chose to conduct the study as a review of reviews and grey literature as a means of efficiency because we were seeking to understand high-level health system features rather than the detail of individual interventions, and because pilot searching suggested that the body of primary studies analysing healthcare access for populations experiencing homelessness was too large for the capacity of the team. Using realist review in this study contributes to the current knowledge base by uncovering causal mechanisms that are at play at the health systems level but which are not measurable and may have been missed or not fully explained in other studies.

The review followed an internal team study protocol based on Pawson's five iterative stages: (1) locating existing theories, (2) searching for evidence, (3) selecting articles, (4) extracting and organising data, and (5) synthesising the evidence and drawing conclusions. ${ }^{28}$ The phases of the review are illustrated in figure 1 .

\section{Initial programme theory}

A rough initial programme theory (IPT) (see online supplemental file 1) was developed based on informal reading and discussions with content experts in the area of homeless health. It listed potentially important contexts (eg, long-term homelessness, regular source of care, competing subsistence needs, knowledge of symptoms and when to seek care) and mechanisms (eg, stigma and discrimination, power dynamic between the provider and the patient, mistrust, fear, feelings of shame/low self-esteem) affecting the outcome of healthcare access for populations experiencing homelessness. The IPT reflected our limited knowledge specifically regarding high-level health systems features that impact healthcare access for populations experiencing homelessness at the outset of the project. It was a way to get into the topic and formed the starting point for shared team knowledge, but it did not turn out to be very useful in directing the further steps in the research, including searching and coding.

\section{Searching}

Iterative searching took place in three rounds. First a systematic search of relevant published peer-reviewed review articles of any method took place. We did not use the IPT in guiding this search as it did not point us in the direction of high-level health system features' impact on healthcare access for homeless populations. Rather, pilot searching suggested that including a number of search terms from the IPT yielded many unrelated articles. Instead, a subject librarian helped design the search strategy, focusing on two broad thematic clusters: homelessness and healthcare access. Further pilot searching was 
undertaken and final search terms were agreed. A formal search of four electronic databases (Ovid MEDLINE, embase.com, CINAHL, ASSIA) was undertaken in April 2019. Results from each search were exported to EndNote V.X9 and deduplicated automatically and manually. Full search terms can be found in online supplemental file 1 .

Additional searching was later undertaken in two rounds. First, citation tracking was used to locate important primary studies included in several of the reviews. At this stage, further peer-reviewed and grey literature sources were included as recommended by the expert panel. Second, a search was conducted to identify additional relevant grey literature sources via the internet and websites of organisations working on homeless health. These sources were selected to shed light on CMOCs that were not fully developed using the data resulting from the systematic search of the peer-reviewed literature and to build additional CMOCs to more fully answer the research question.

\section{Selection and appraisal of documents}

Studies were included based on their relevance to the review question, that is, if they provided information about access to healthcare for homeless populations and would contribute to the development and refinement of programme theory. Only studies in English were included. RS screened all titles and abstracts and SB independently screened a random $10 \%$ sample. During the screening phase an initial evaluation of rigour and relevance (see glossary in online supplemental file 1 for definitions) was done, and as was the case at later stages when additional literature was collected. See full search in figure 2.

\section{Data extraction and analysis}

After the screening of the peer-reviewed literature was completed, a summary table was created listing the key features of each study (table 1 ).

The first round of coding took place in NVivo V.12 of the peer-reviewed articles from the systematic search, starting with the articles deemed most useful regarding rigour, relevance and trustworthiness (eg, articles that had rich data and had adhered to their chosen research methodology), as they were expected to best inform the development of CMOCs and programme theory. Coding was first done inductively with useful pieces of data assigned to thematic codes created by the researcher one by one through the coding process. Codes were then organised into groups depending on whether they were related to the individual seeking care, the healthcare practitioner and/or programme providing care, or the health service/health system in which that healthcare is organised. Labels of context, mechanism and outcome were also assigned when possible and at times not assigned if a code did not have a clear placement in a potential configuration. The rest of the peer-reviewed articles were coded deductively using the codes already created, with more codes inductively added as needed.
Using a realist logic of analysis, CMOCs were then created from the data in the review articles included after the first round of searching, drawing from several sources to construct each CMOC. The purpose of the analysis was to identify general patterns of outcomes which can be expected to occur when mechanisms are activated in the right context, with some regularity (also called 'demiregularities'). In other words, the analysis identified outcomes which regularly, but not always and not in every case, result from the causal processes described in each CMOC discussed later.

The crafting of CMOCs took place by sorting codes from NVivo according to whether they mainly belonged to contextual factors, represented an outcome or whether they were mechanistic in nature. We then, through constant consultation of the literature, linked contexts, mechanisms and outcomes that fit together to explain underlying causation. We wrote a list of resulting CMOCs that all demonstrated a causative relationship affecting healthcare access for populations experiencing homelessness, as described in the data. This list of CMOCs was written in a Word document with its supporting data excerpts listed. Iterative cycles of analysis then took place to refine, challenge and change the CMOCs with the data. Through the analysis process, some CMOCs were eliminated and some were combined if they were similar to others.

The CMOCs generated from the data extracted from the peer-reviewed literature were further refined after a formal expert panel meeting, with representation by members of an inclusion health team in a local hospital (consultant doctor and social worker), the director of policy for a national homeless charity, an inclusion health general practitioner (GP), a representative of the Health Service Executive, and two academics who work in the areas of homeless healthcare and health policy. The expert panel discussion led to the strengthening of the research in several key ways, with the following two being the most important: first, a discussion of the diversity within populations experiencing homelessness resulted in refining the focus of CMOCs to outcomes relating to the specific homeless subpopulation of single adults experiencing long-term homelessness and complex needs; and second, discussions revealed that sufficient data about a number of important health system features which impact access, for example, financing, governance, organisational culture, policies and strategies, were missing from the peer-reviewed literature sources and various suggestions were made for sources that would inform that shortcoming.

To attempt to more fully explain high-level health system features which impact access, additional data were collected then through citation tracking and through additional searching of grey literature sources. The results of these searches were screened and then coded in a similar way to the peer-reviewed literature. Initial codes were developed inductively from the first several sources and then applied deductively, with new codes created as 

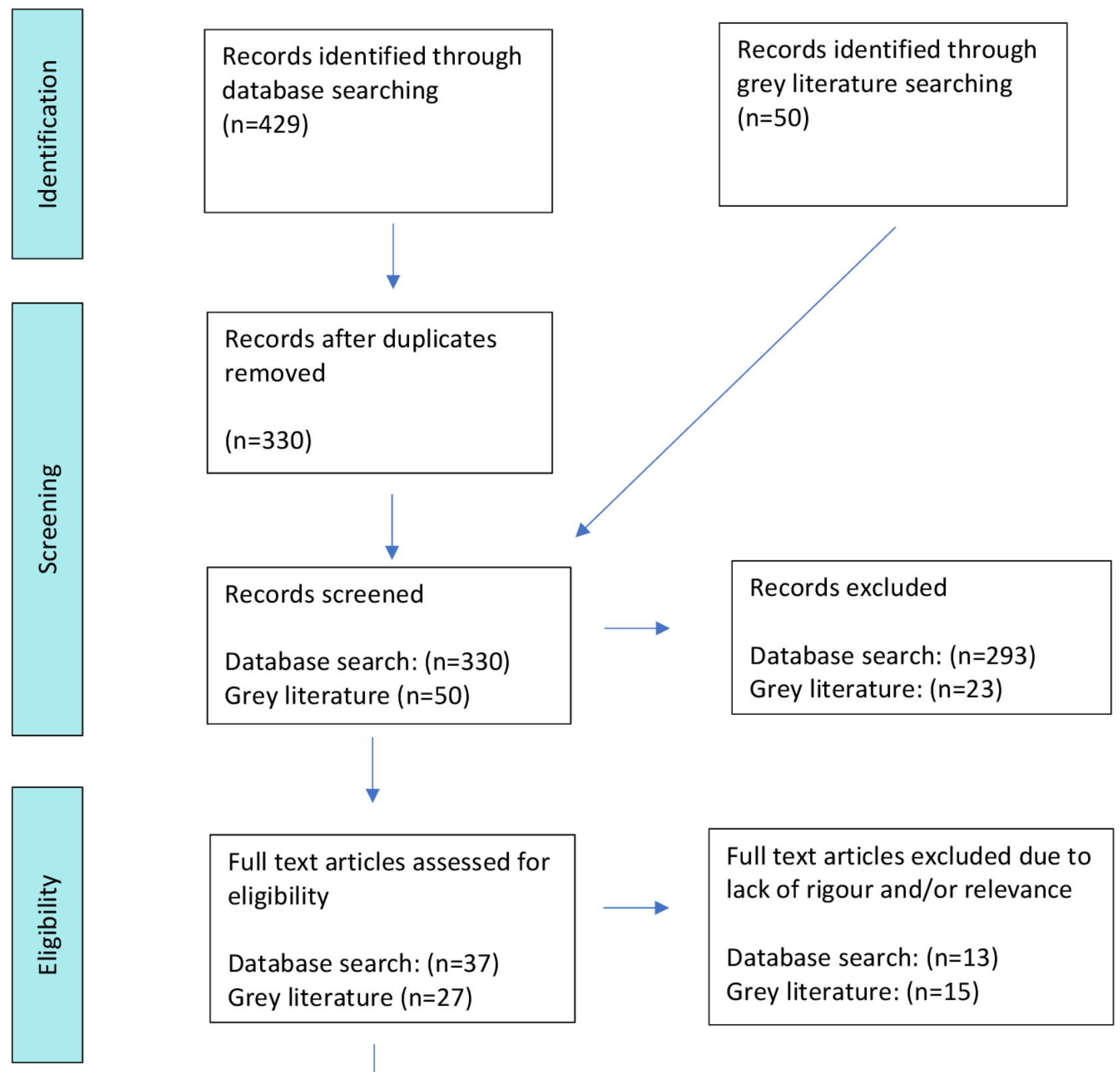

Full text articles assessed for eligibility

Database search: $(n=37)$ Grey literature $(n=27)$

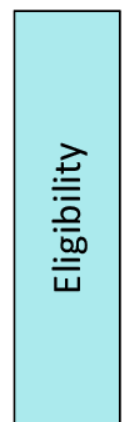
Studies included in synthesis
Database search: $(n=24)$
Grey literature: $(n=12)$
Expert/citation tracking $(n=11)$
Total: $(n=47)$
Full text articles excluded due to lack of rigour and/or relevance
Database search: $(n=13)$
Grey literature: $(n=15)$

Figure 2 PRISMA diagram.

needed as the remainder of the sources were coded. Labels of context, mechanism and outcome were assigned when possible and at times not assigned if a code did not have a clear placement. Contexts, mechanisms and outcomes are not static labels, but can change in relation to each other; for example, something can be a context in one instance but a mechanism in another, and an outcome can become a context for another CMOC depending on its explanatory role in the given configuration. ${ }^{30}$ Codes were then organised into groups depending on 


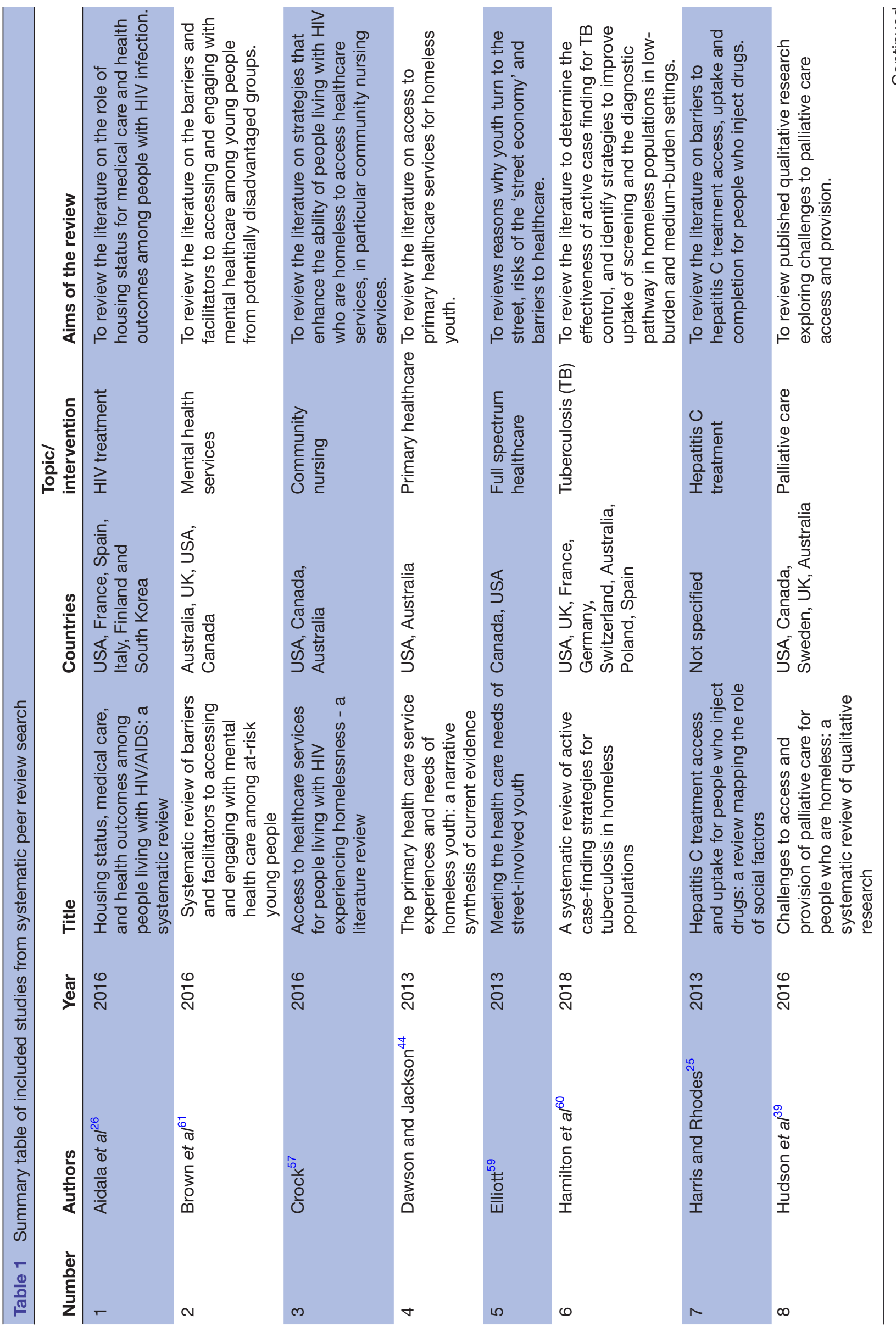

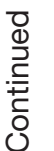




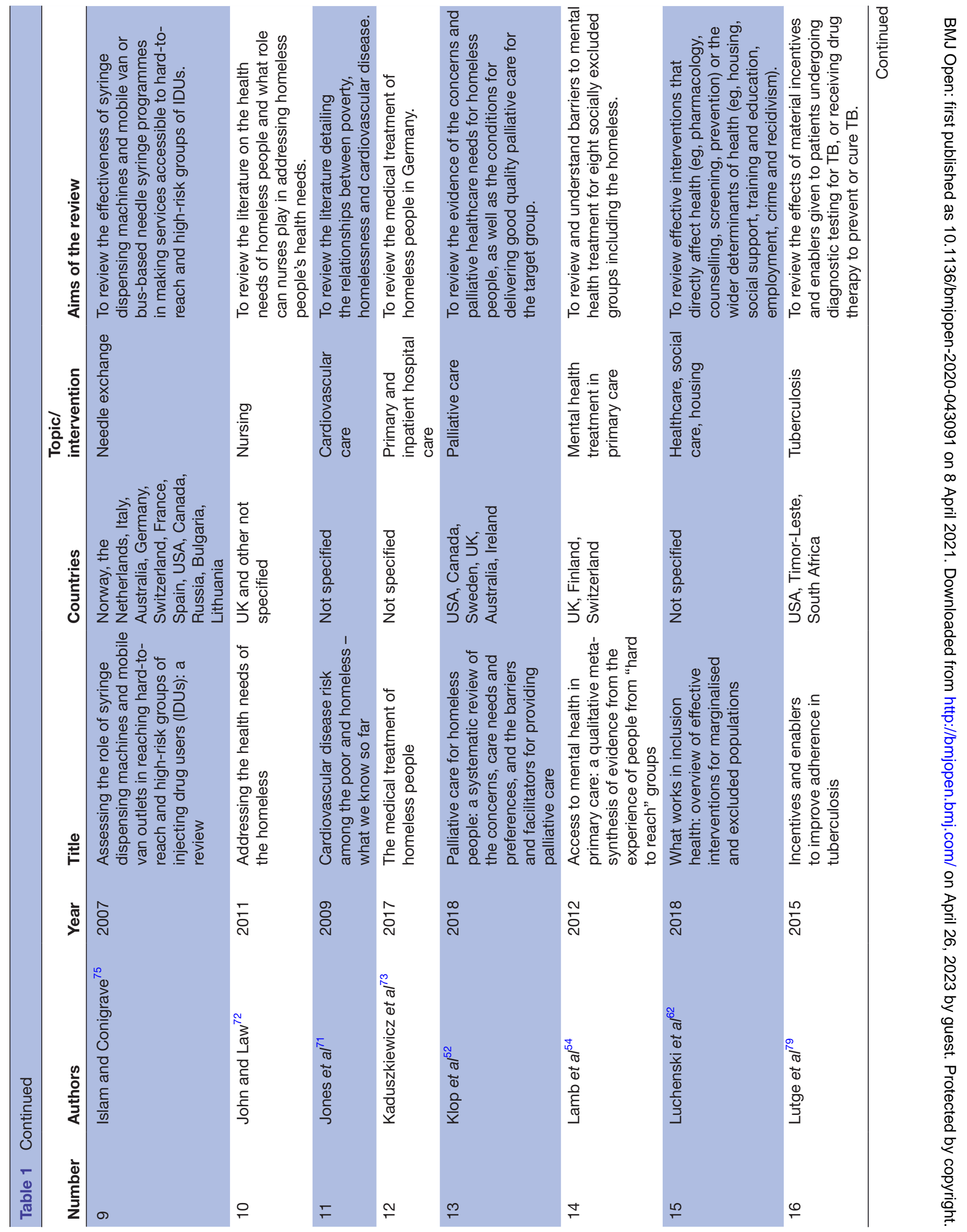




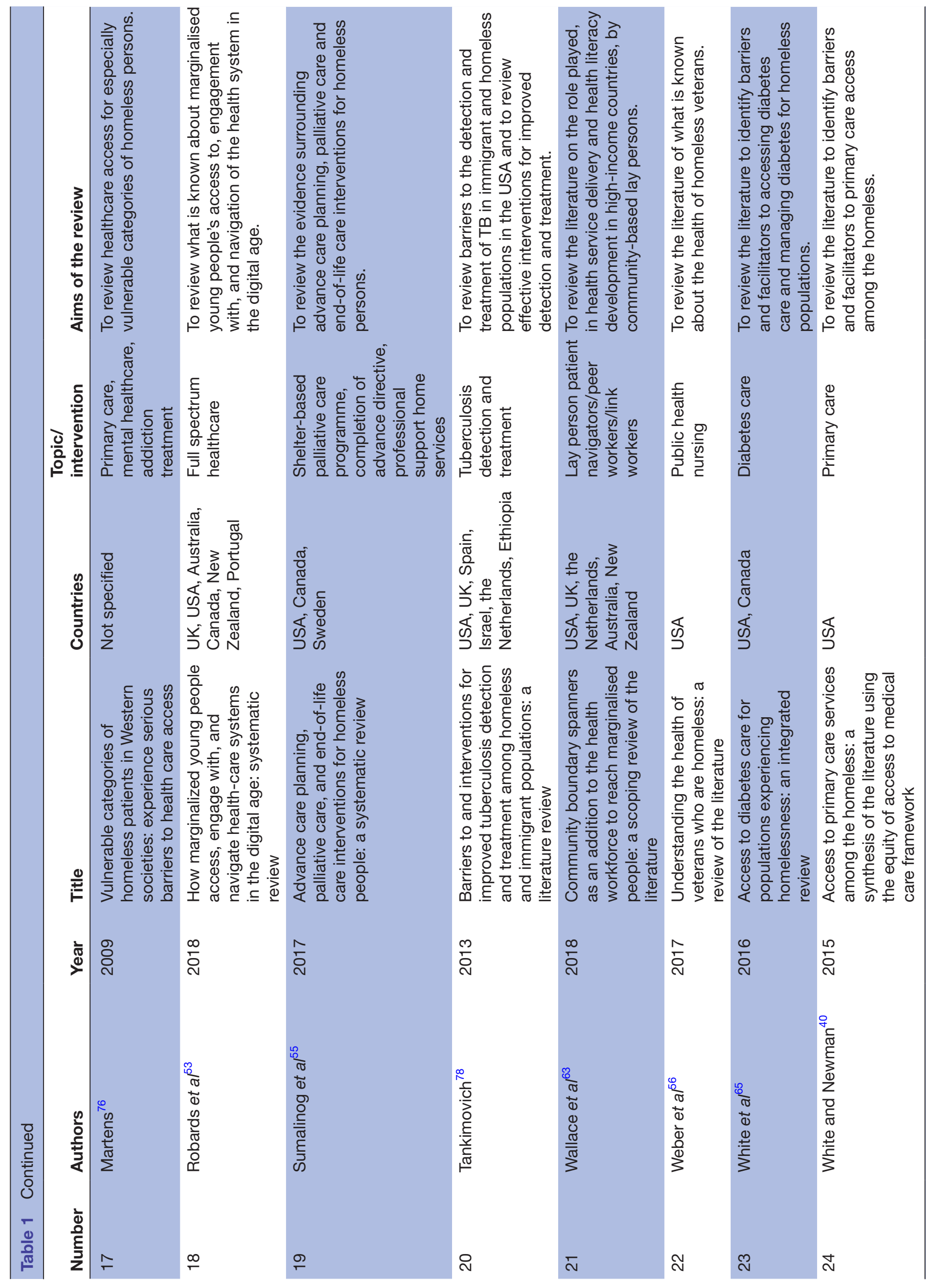

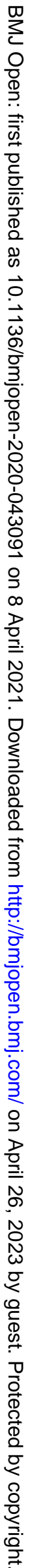


whether they were related to the individual seeking care, the healthcare provider and/or programme providing care, or the health service/health system in which that healthcare is organised. These new data were then used to create more CMOCs using the new data combined with those collected from the first round of searching and to further refine the existing set created from peer review data where useful.

\section{Patient and public involvement}

No patients or members of the public were involved in this research.

\section{RESULTS}

\section{Search results and study characteristics}

In total, 330 titles and abstracts were screened and 37 published review articles were deemed to meet the inclusion criteria and these then underwent full-text review. Next, during a close reading of each full article, it was determined that 13 of the 37 articles that were initially included in the analysis did not fully meet relevance and/ or rigour standards and they were thus excluded, leaving a total of 24 articles for inclusion (see table 1). All studies included in the reviews were from high-income countries, the majority having focused on the USA, Australia and the UK.

An additional 23 primary peer-reviewed articles and grey literature sources were identified through citation tracking, from expert recommendations and through a subsequent purposive search of the grey literature via Google and the websites of relevant organisations. The majority of these were from the UK.

\section{Focus of the review}

Initial analysis resulted in two broad conceptual sets of findings: one related to how services are organised and delivered, and another related to the process of training and the development of the right professional skills, attitude and awareness which allow the staff to best provide such services. Based on team discussions and with the recognition that we would not be able to fully explore all findings, we decided to focus on the topics most relevant to the research question. The findings around service delivery and organisation were chosen as the focus for the next stage of the review.

\section{Programme theories and $\mathrm{CMOCs}$}

Using the data collected from the three rounds of literature searching, a total of 73 individual CMOCs were generated through several rounds of analysis over the course of several months. There was overlap among some of the CMOCs, but it was important to capture and articulate the relationship between each distinct context, mechanism and outcome and to interrogate whether they shared a causal relationship before combining some together and rejecting others.

Once that analysis was completed, we grouped together similar CMOCs into the six consolidated CMOCs as described in detail in the following narrative.

In the CMOCs, while the contexts operate on a health system or service level, mechanisms are activated within individuals (eg, patients, healthcare practitioners, staff), which makes intuitive sense as a mechanism 'involves the interaction between particular inputs (or resources) and human reasoning, which produces a particular outcome (or not) ${ }^{31}$

\section{CMOC 1: resourcing}

The first consolidated CMOC (figure 3) explores contexts where mainstream healthcare practitioners and staff (in hospital and primary care settings) are expected to treat a high need patient group (long-term homeless populations with complex needs) but are not provided adequate resources and incentives (eg, extra funding for longer appointments and more coordination), while also having inadequate expertise in the particular needs and life experiences of this group. ${ }^{32-35}$ In these contexts, practitioners and staff feel that servicing patients experiencing
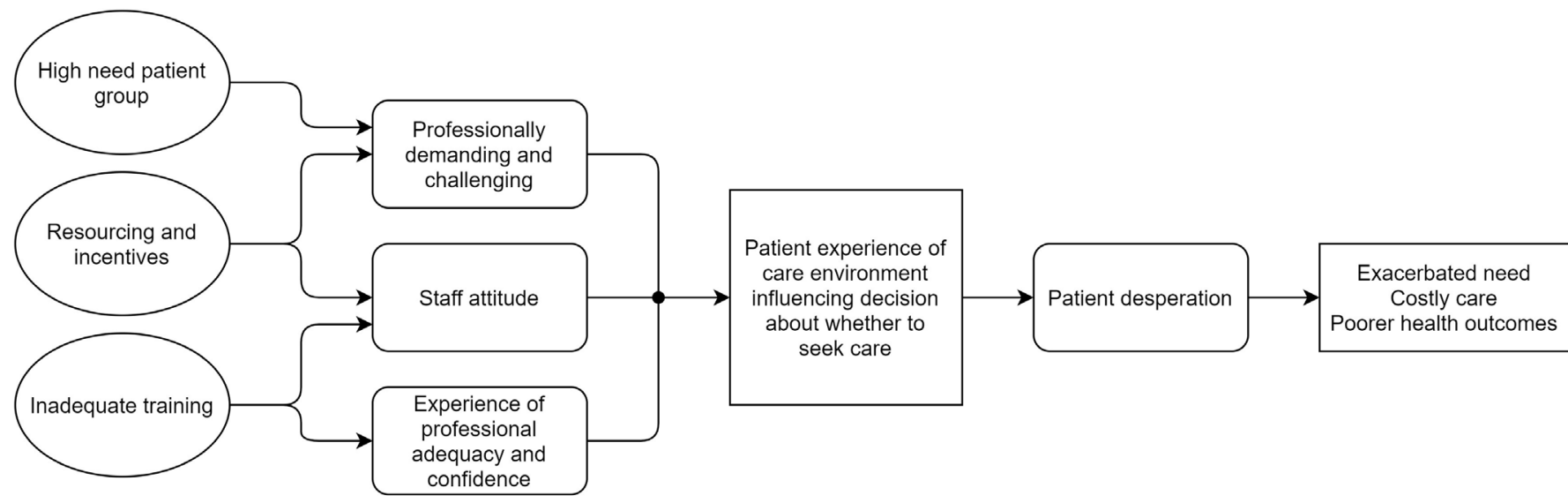

C

$\mathrm{M}$

$\mathrm{O} / \mathrm{C}$

M

Figure 3 CMOC 1: resourcing. CMOC, context-mechanism-outcome configuration. 


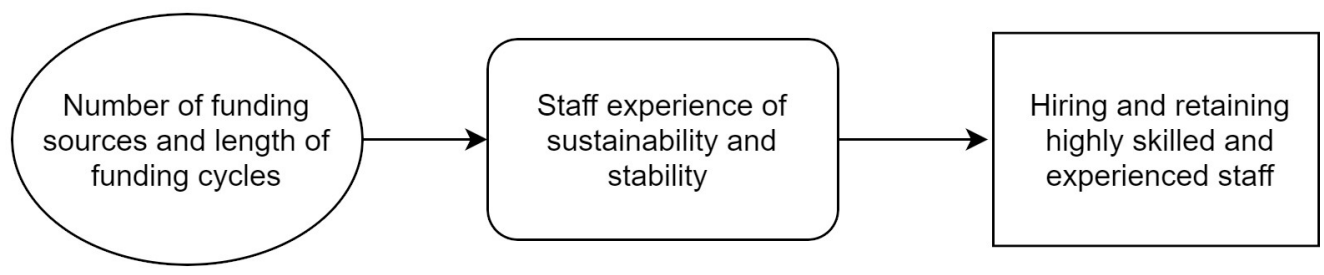

C M $\quad 0$

Figure 4 CMOC 2: funding stability. CMOC, context-mechanism-outcome configuration.

homelessness is professionally demanding and challenging. They experience feelings of professional inadequacy and lack of confidence and display an unwelcoming attitude towards patients. ${ }^{172-34} 36-40$ As a result, patients' experience of the care environment is a negative one, which causes them to choose not to seek care at an appropriate time (eg, seeking care from a GP before a condition gets worse and acute care is needed). ${ }^{17323741-43}$

The outcome of not seeking care when the care environment is not welcoming becomes a new context which leaves patients instead to seek care only when the need is emergent and out of desperation, and at a later stage than ideal and at a setting that is readily available (such as the ED). ${ }^{1737394144}$ This results in exacerbated need, leading to more complex, intensive and costly care, which ultimately generates poorer health outcomes. ${ }^{374} 45$

\section{CMOC 2: funding stability and source}

CMOC 2 (figure 4) shows that in a context where funding for health services comes from multiple sources and where funding cycles are short and unreliable, for example, grant funding for specific purposes with a short duration, ${ }^{38414346}$ staff members employed on short-term contracts experience a lack of sense of stability and sustainability because they are in a series of continual contract renewals and do not have job security and, especially in periods of austerity, may experience wage cuts and wage freezes. The precarious sustainability of the services they work for leads to staff being asked to do more with less. Work tasks change when pilot schemes and new initiatives bring additional goals tied to new funding while staff still have to complete existing tasks. Additionally, individuals working in these services are often seen by their managers as being naturally caring people who are intrinsically motivated to 'do good' and who do not need adequate pay and conditions because of the perception that their motivation lies elsewhere. ${ }^{35434-49}$ Frequent changes make it difficult to achieve good outcomes for people who need extra time and attention and for whom forming trusting relationships with practitioners is particularly important in accessing care, as is explored in CMOC 5. The outcome is difficulties hiring and retaining highly skilled and experienced staff members. ${ }^{35} 4347-49$

\section{CMOC 3: health system fragmentation and goals}

CMOC 3 (figure 5) shows that in a context where various parts of a fragmented health system operate in silos with a narrowly defined scope of goals, ${ }^{3738414350}$ staff engage in organisation-centred thinking and prioritise the goals of the health system even when they are not suitable for responding to the complex needs of homeless patients whose care should be organised across a number of domains. Staff often lack knowledge of how to best meet the needs of patients experiencing homelessness and complex needs, while health system goals and targets are more clearly defined as part of professional structures and workstreams. ${ }^{37} 3850$ Staff and practitioners are not incentivised or empowered to take responsibility for creating holistic, coordinated and flexible ways of organising care around a patient's needs and wishes because they are instead asked to meet narrowly defined targets tied to funding and the expectations of commissioners. As a result, healthcare is organised around explicit and implicit health system goals, not the needs or goals of the person. ${ }^{183738415051}$

\section{CMOC 4: care organised around the person}

In contrast to CMOC 3, CMOC 4 (figure 6) shows that when care is organised around the person and their needs, resulting outcomes produce conditions that

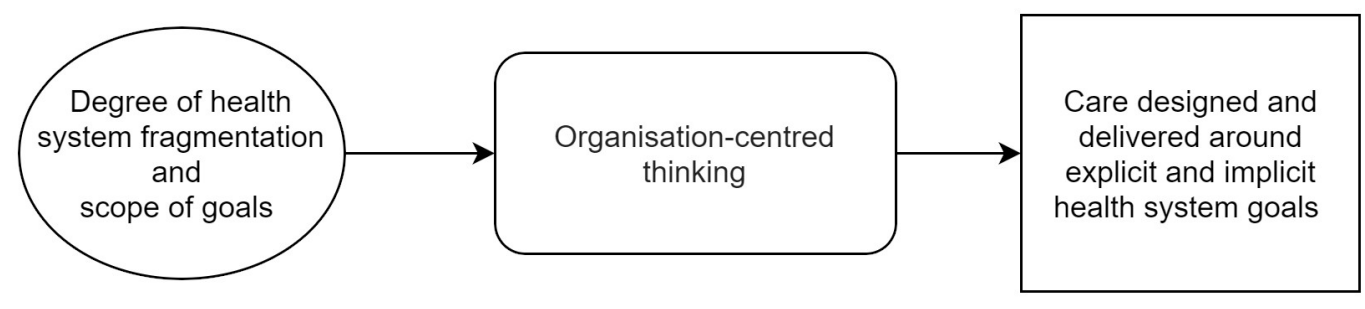

C

M

O

Figure 5 CMOC 3: fragmentation and goals. CMOC, context-mechanism-outcome configuration. 


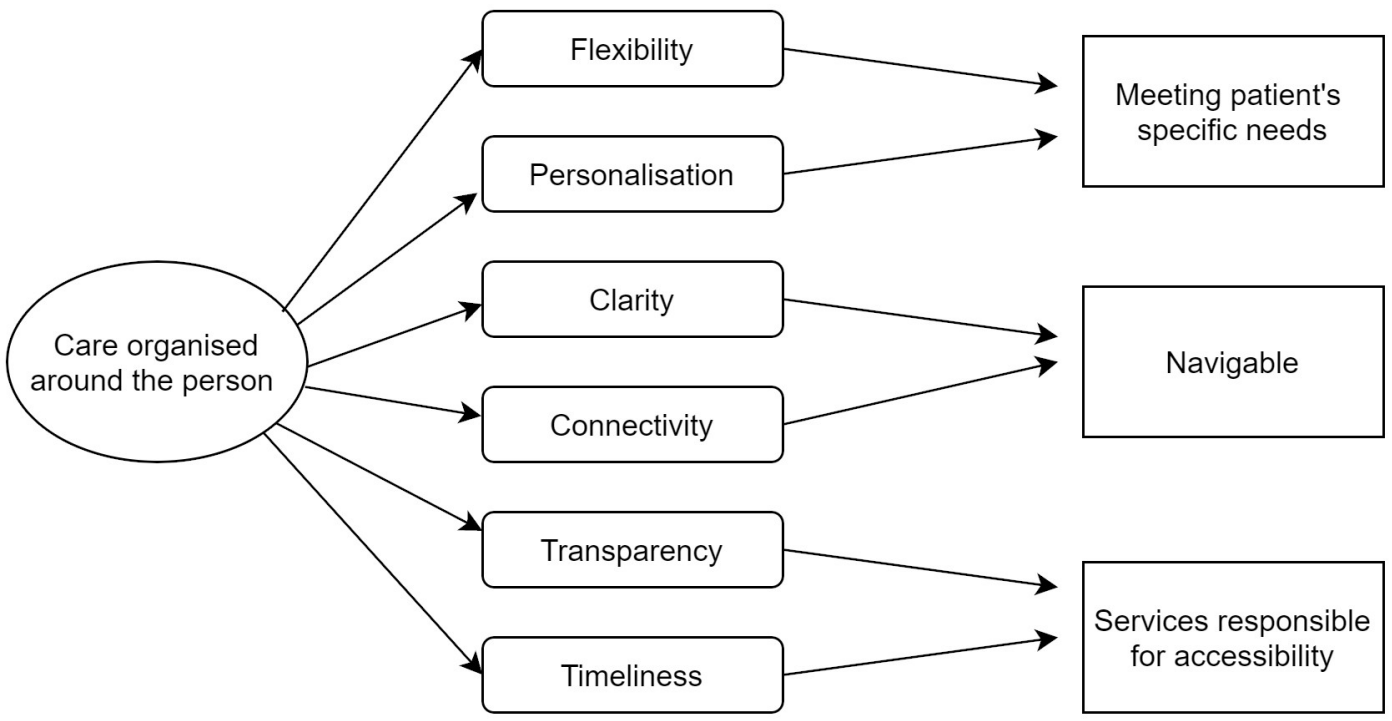

C $M$

$\mathrm{O}$

Figure $6 \mathrm{CMOC}$ 4: care organised around the person. CMOC, context-mechanism-outcome configuration.

promote healthcare accessibility. In this context, the mechanism of flexibility and personalisation is triggered, allowing patients to feel that their particular needs are understood and met. $2535395052-55$

Additionally, health system navigability is enhanced through having coordinated and co-located care, ${ }^{735} 39405356-58$ through having clear and intuitive patient pathways managed by staff and practitioners who create connectivity between services by bringing patients along to any subsequent treatment, scan, consultation and so on in the given patient pathway, ${ }^{189535960}$ and through accompanying patients either by link workers or peer advocates. ${ }^{25} 3960-64$ Having this kind of support helps patients by providing extra motivation and confidence in seeking health services when needed, navigating institutional locations such as hospitals and clinics, and negotiating patient pathways which may be complex. ${ }^{39} 415360$ Peer advocates have a unique understanding of the experience the patient is having and have the ability to meet patients where they are and provide emotional and practical support. ${ }^{39} 445861-63$

Finally, when care is organised around the person, it is inherently respectful of other demands on their time and their particular goals (or lack thereof) for their own health. Services are characterised by timeliness and openness. For example, if as many services as possible are carried out in one clinical encounter and a course of treatment is chosen through shared and transparent decision making, then responsibility is placed on the service to make itself fit with the patient's life circumstances and to share knowledge and decision making to promote initial accessibility and beyond. ${ }^{25} 395759-6265$
CMOC 5: inclusive culture and leadership

CMOC 5 (figure 7) examines the importance of culture and leadership in creating inclusive health system contexts. Through the dominant culture the value assigned to people from stigmatised groups contributes to their experience of poorer care because of prejudice and stereotypes commonly held about them. Populations experiencing homelessness face stigma when engaging with all areas of society, including healthcare settings. ${ }^{25} 52626667$ Creating the right cultural context depends on leadership and management through explicit commitments to values of inclusivity and whole organisation policies and processes. $333741435168-70$

In the articles reviewed, stigma is a crucial mechanism which keeps individuals experiencing homelessness from accessing healthcare ${ }^{1718253539586171}$ (the impact of stigma on the patient's experience is explored in CMOC 1). On the other hand, when individuals experience an environment that is non-stigmatising, respectful, empathetic, accepting, sensitive and understanding of their life experience and particular needs, trust develops between the patient and the practitioner(s) and a positive feedback loop is created, leading to a productive patient-practitioner relationship developing over time. Still operating in a context which explicitly values and promotes inclusivity, trust becomes a reinforcing mechanism whereby deepening trust facilitates ongoing engagement, which leads ultimately to ongoing appropriate access to health services at the right time and right place. ${ }^{39} 4052-545761-63$

\section{CMOC 6: flexible healthcare delivery}

CMOC 6 (figure 8) explores the context of flexibility in healthcare services delivery in terms of appointment length, availability of walk-in appointments and 


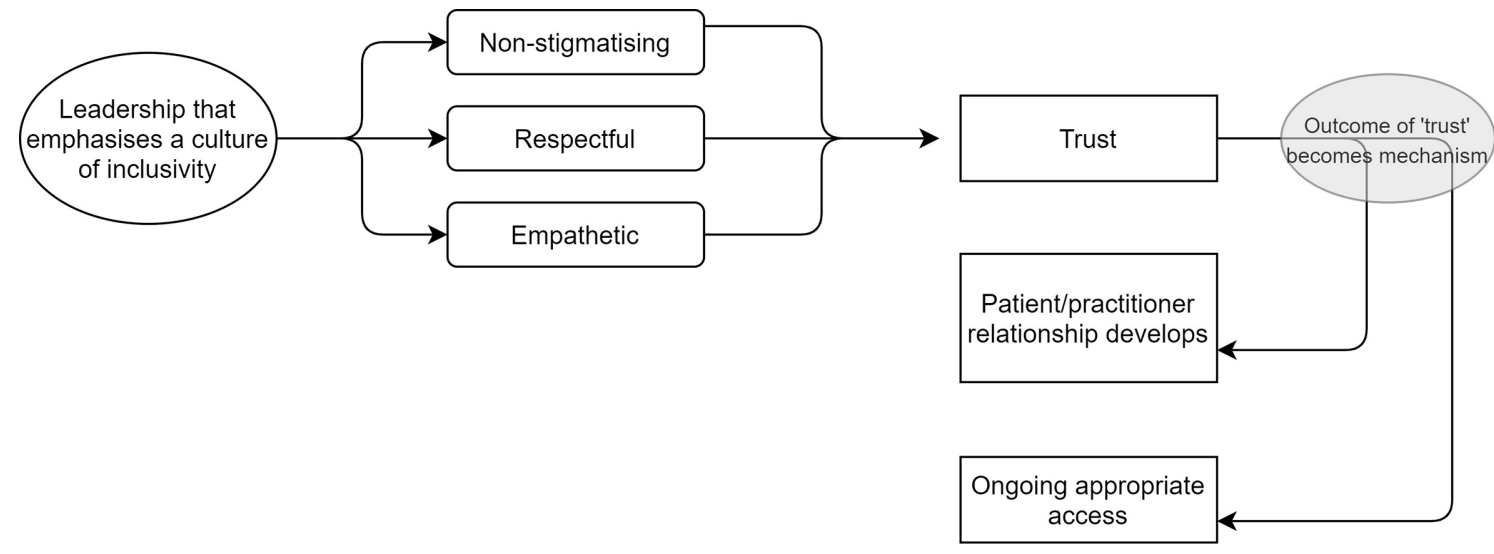

C M $O$

Figure 7 CMOC 5: inclusive culture and leadership. CMOC, context-mechanism-outcome configuration.

self-referral, opportunistic add-on services such as vaccinations and screenings, allowing dogs, trauma-informed practice and understanding of behaviours that trauma can result in, and more. ${ }^{2532434652535657596172-74}$ Flexibility is also a mechanism in CMOC 4, while here it is acting as a context characterising the approach to and design of care delivery.

In this context, practitioners and staff with expertise and experience with the population group are able to anticipate the common interventions that may be needed, to adapt to the particular needs of the patient in front of them, and to use their expertise to provide the treatment that is most needed in the current situation. Communication is tailored to the patient with awareness of potential literacy issues common in the population. 394044545960626575 This stands in contrast to what happens when services do not have proper resources and when providers and staff do not have expertise and experience as explored in CMOC 1.

In the context of flexible healthcare delivery, patients' needs are identified, and if met in the manner described in CMOC 4 this experience forms a new context. In this new context ('needs identified'), experiences of lowered frustration and fear are engendered in patients because they do not have to fit into a mould of a health service which is difficult to navigate and not likely designed with them in mind..$^{40} 42565761$ Resulting from that, patients feel seen and understood, which becomes a new context

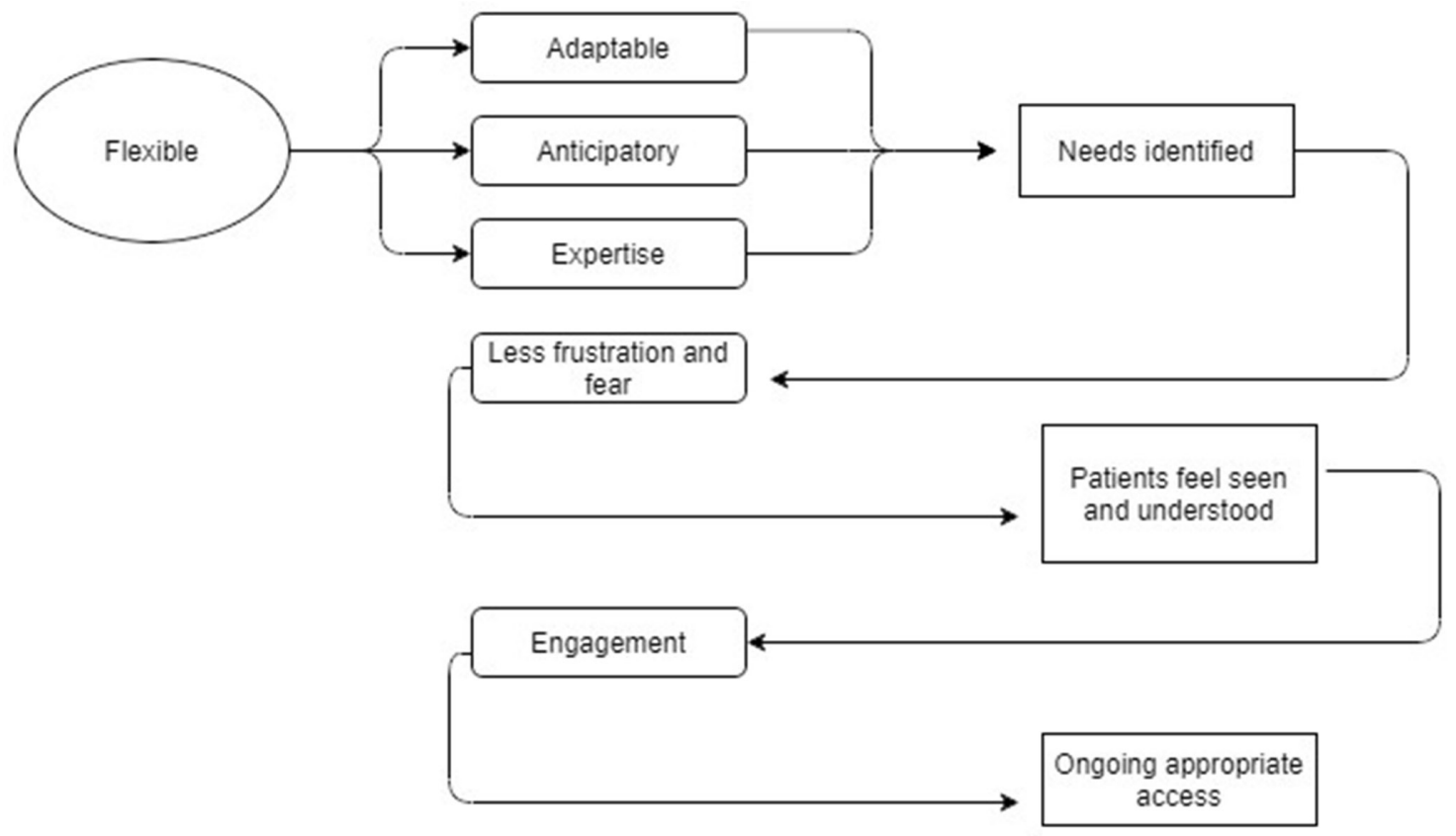

Figure 8 CMOC 6: flexible healthcare delivery. CMOC, context-mechanism-outcome configuration. 


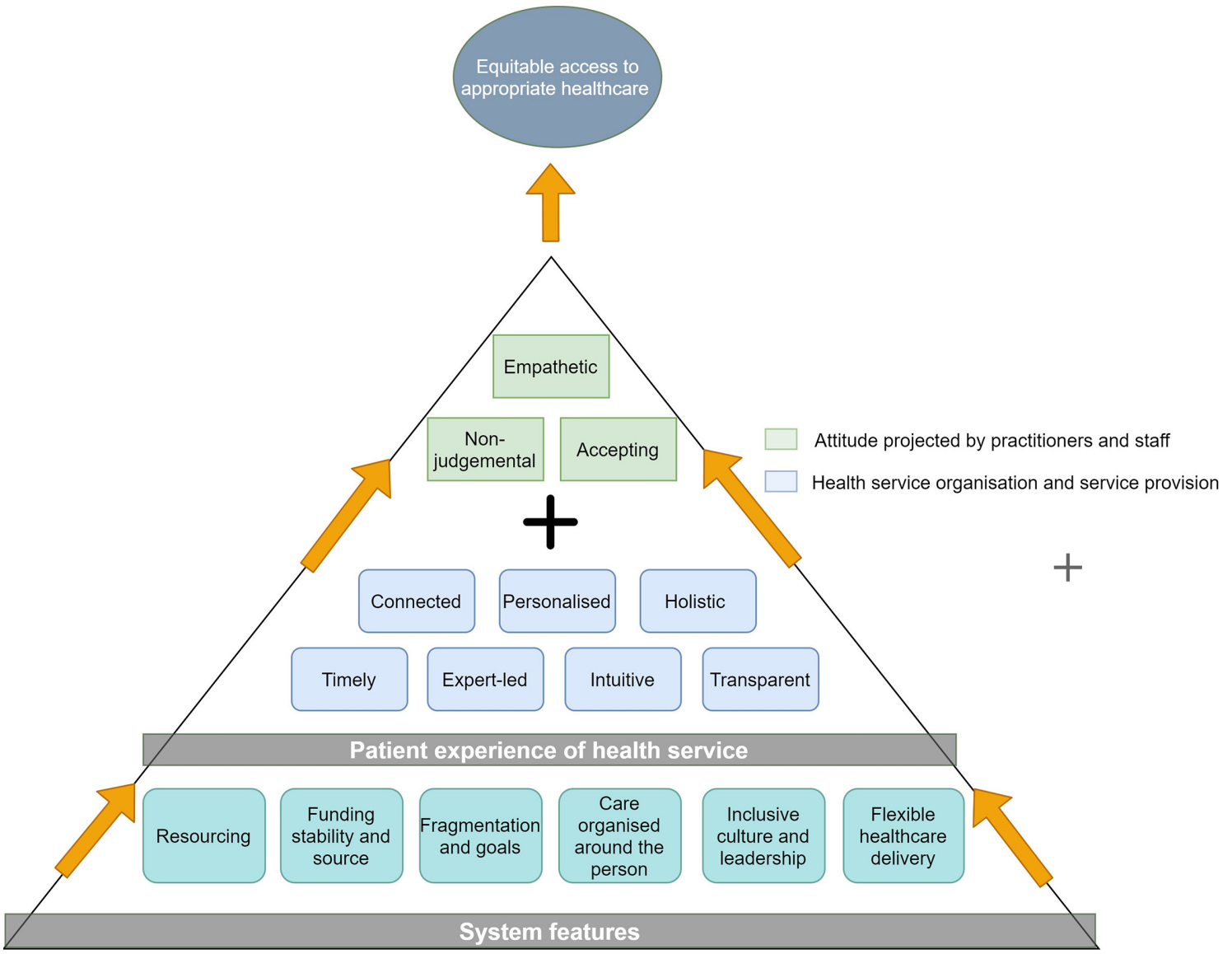

Figure 9 Overarching programme theory.

('patients feel seen and understood') in which ongoing engagement from both sides is possible and pathways can be created with high levels of flexibility and assistance. $^{44} 52-5461$ Patients are able to follow these peer, key worker and/or health staff assisted pathways, and ultimately this leads to ongoing appropriate access to services over a course of treatment or on an ongoing basis as needed. A cyclical nature of an ongoing and trusting relationship is established and reinforced over multiple interactions. ${ }^{25} 394461-637276$

\section{Full programme theory}

The overarching programme theory synthesising the full set of findings from this review (figure 9) shows that a set of interlinking factors must all be in place for healthcare access to be successful for populations experiencing homelessness.

It shows that the combination of resourcing, funding stability, health system fragmentation and goals, the degree to which care is organised around the person, the degree to which leadership promotes an inclusive culture, and the flexibility of healthcare delivery set the stage for accessibility.

When resources (funding, expertise, experience of patient group) are adequate, when funding cycles are long and stable, when there is a low degree of fragmentation and health service goals support integrated working, when healthcare is organised around the patient and delivered flexibly, and when services are provided in an inclusive culture championed by leadership, then services can be provided in the way explained in the blue and green boxes in figure 9. That is that healthcare services are organised and provided (as explained in the blue boxes) in a manner that is connected to and collaborating with other practitioners and services; personalised to the needs and desires of the patient; holistic in looking at the patient as a full person; timely in that services are available when needed and take into account the patient's lifestyle and other commitments; expert-led where staff and practitioners have adequate expertise and experience of populations experiencing homelessness, allowing them to anticipate and opportunistically act on multiple healthcare needs in one encounter; intuitive in that patients can understand and easily follow patient pathways, with staff/peer advocate assistance as needed; and transparent in that confidentiality is clear and decisions are made between the patient and the provider in an open and honest way based on the patient's desires and not the needs of practitioners. Additionally in an inclusive culture with flexibility and autonomy allowed, staff with the right experience and expertise are enabled to project an attitude of non-judgement, acceptance and empathy, as described in the green boxes.

On the other hand, when those system features are not in place or not fully in place, the patient experience of 
health services is impacted negatively. For example, when health services are provided in a fragmented way, it is not possible to provide timely care because each service operates on its own schedule and each step on a patient pathway depends on a referral from the last.

\section{DISCUSSION}

\section{Statement of principal findings}

Health systems influence healthcare accessibility for populations experiencing homelessness in a number of ways as described in the six CMOCs explained. The analysis has shown the following:

- When healthcare practitioners have limited experience, training and resources to meet the particular needs of populations experiencing homelessness, they feel professionally inadequate and project unwelcoming attitudes. As a result, patients have a poor experience in the care environment and choose not to seek care until they experience their situation as desperate, resulting in exacerbated need and poorer outcomes to follow.

- When health services have short funding cycles and diverse sources of income, staff and practitioners perceive their employment situation to be unstable and unsustainable, and as a result hiring and retaining qualified and experienced staff are jeopardised.

- When health systems are fragmented and operate in silos with a narrowly defined scope of goals, practitioners and staff focus on and seek to meet the goals of the organisation they work for rather than those of the individual patient.

- Conversely, when care is organised around the person, it can be provided in a manner that is personalised and flexible, which results in meeting the particular needs of the patient; services work together connecting around the patient's needs and providing the patient with clarity of what their next steps are and give assistance in reaching those next steps, leading to the patient journey being easier to navigate; and services take responsibility for accessibility by seeking to understand the full needs of the patient, respecting their time and other commitments, and being transparent about the reasons why a given treatment is needed.

- When healthcare leadership and management support and champion inclusive cultures, it allows services to be delivered in a non-stigmatising, respectful and empathetic manner, which leads to trust developing between patients and staff. Over time, the experience of trust in a context of an inclusive culture reinforces and strengthens the patient-practitioner relationship and ultimately allows for ongoing healthcare accessibility.

- When health services are delivered in a flexible manner, practitioners can use their expertise to anticipate, and adapt care to, the needs of the patient. As a result, the patient's needs are identified, and if met the patient experiences less frustration and fear, which leads them to feel seen and understood, which promotes ongoing engagement with services and ultimately leads to ongoing access taking place.

Making healthcare accessible for populations experiencing homelessness who have complex needs requires stable funding for sustainable staffing, coordination of services which should be delivered in an inclusive culture championed by leadership, and space to allow practitioners and staff to use their expertise to provide anticipatory, flexible care. Trust and the development of ongoing trusting relationships between patients and staff are key.

\section{Strengths and limitations}

In this review we have used the RAMESES publication standards ${ }^{77}$ to guide us in rigorously and systematically reviewing and synthesising the literature included here.

This analysis has taken a high-level view of systematic factors across a breadth of data not focused on specific interventions or disease areas. Our findings have uncovered modifiable health system and service contexts that are applicable and transferrable among high-income countries. The analysis has been informed by the involvement of content experts and stakeholders who have confirmed and challenged findings resulting in further refinement.

Like any review this one relies on the available literature. A potential limitation of this study is that the initial search of peer-reviewed articles included only literature reviews, which may mean that we missed some articles. However, a large volume of relevant data were identified through the reviews and these were further augmented by citation tracking, expert advice and additional searching to identify additional key documents. Our search did not identify studies from low-income and middle-income countries and we cannot therefore say whether our findings are transferrable in such contexts. More research is needed to explore how the contexts outlined here are applicable or not to low-income and middle-income countries, how, for whom and why.

Much like a majority of the literature on access to healthcare for populations experiencing homelessness, the IPT took the perspective of an individual seeking to access healthcare services. However, as the goal of this review was to explain health system factors impacting healthcare access for populations experiencing homelessness, the IPT did not provide a helpful framing for the goals of the study and we did not use it in the search, data extraction or analysis phases as is often done in realist work.

This review did not use patient and public involvement (PPI). The research team felt that PPI would not have added sufficiently to this system-level analysis to warrant the use of potential participants' time and resources. Furthermore, we felt that appropriate 'PPI-like' engagement for a study like this should be with front-line staff who function at the intersection of the patient experience with health system goals, culture and operational procedures. To capture this perspective, we held a formal 
expert panel meeting with a number of stakeholders representing professional groups working with populations experiencing homelessness. More similar engagement during the design phase of the study would likely have been helpful and could have added to the IPT building, searching and analysis phases of the study.

None of the included peer review studies specifically analysed health system features. Some analysed service/ programme-level features that promote or impede access, ${ }^{403}$ but the majority of reviews synthesised and described the accessibility of particular individual-level treatments such as for tuberculosis, diabetes, palliative care and cardiovascular disease. ${ }^{39} 526065717879$ However, grey literature sources added substantially to the set of findings about high-level health system contexts and mechanisms (eg, resourcing, expertise, funding cycles, fragmentation and goal setting).

The included data sources did not differentiate between health access outcomes specifically in response to health system features within subgroups among long-term single homeless individuals with complex needs. Therefore the 'for whom' part of the analysis was not as fully developed as it might have been, with more research needed in this area.

There was wide variety in the quality of the reviews themselves, with some being transparent and following the guidelines of their particular review methodology closely (eg, search string and list of databases provided, appropriate quality appraisal tools used, systematic approach, using appropriate reporting standards, and so on), while others did so to varying degrees and a few not at all. A majority of reviews were based on qualitative, descriptive and small-scale quantitative studies using a variety of methods, for example, randomised controlled trial, cohort and case-control studies. The majority of the included studies evaluated a particular aspect of healthcare access, for example, association between having health insurance and having a usual source of care. ${ }^{80}$

\section{Comparisons with existing literature}

We are aware of no other realist reviews examining healthcare accessibility for populations experiencing long-term homelessness and complex needs and of no other reviews of any approach that have examined high-level health system features that impact healthcare accessibility for the same population group. Other reviews have focused on specific healthcare interventions and most take the point of view of the patient navigating services or systems. This review differs in that we have analysed the upstream health system and service contexts that promote healthcare accessibility and have found generalisable features not specific to a particular intervention or the treatment of particular illnesses. These uncover causative relationships between how healthcare is organised and delivered at a systems and service level and its accessibility for populations experiencing homelessness and complex needs.

A realist review by Ford $e t a l^{81}$ of factors that impact access to primary care for socioeconomically disadvantaged older people in rural areas focused on the patient journey in accessing healthcare and not on health system features. However, there were similarities between its findings and ours: at the service level, both studies found barriers related to ease of booking, clarity of information, system and service navigability, provider responsiveness to patient needs, patient empowerment, social status, trust, and clinician empathy and capacity.

There are similarities between our review and Aday and Andersen's 'A framework for the study of access to medical care'. ${ }^{12}$ Our CMOCs all explain aspects of the two first boxes in their framework: health policy (eg, financing, education, manpower and organisation) and characteristics of health delivery system (eg, resources, organisation).

Our CMOCs also hold some similarities with Levesque et $a$ 's 'Patient-centred access to health care: Conceptualising access at the interface of health systems and populations, ${ }^{82}$ although it is focused on the individual patient journey. Concepts including acceptability (eg, professional values, norms and culture) as well as appropriateness (eg, technical and interpersonal quality, coordination and continuity) are important to CMOC 1, 4, 5 and 6 .

\section{Meaning of the study}

As in other realist works, our analysis has uncovered demi-regular patterns of outcomes resulting from mechanisms being activated in the specific contexts described in the literature. Mechanisms are not always activated in a given context. Outcomes are occurring both at a system level and an individual level. The topic of access to healthcare for homeless populations is a complex one in and of itself, but it sits within an area of much more complexity: the general treatment of homeless and socially excluded populations in all areas of society including health, housing and social care. The narrow focus on access to healthcare here is due to the specificity needed to conduct sound research; however, this focus is not meant to be seen as an argument for siloed thinking and service provision.

In fact, population groups on the extreme margins of society, such as populations experiencing long-term homelessness, are in particular need of comprehensive, joined-up strategies in their care. These populations tend to exist at the intersection of multiple disadvantages in life (eg, substance use, childhood trauma, poor mental health, contact with criminal justice systems), which can amplify each other. At the same time, these people also have a high risk of falling into gaps between service and policy areas each of which are focused on their particular goals and targets. For example, housing and health policies are often not joined up. ${ }^{83}$

It is well known that lacking adequate housing in and of itself causes illness to occur. ${ }^{2}$ It is likely that many of the findings in this review can be applied more broadly to all socially excluded populations and form a basis for thinking about how to make a health system fully 
responsive to populations experiencing a variety of deprivation or differentiation from 'the norm' and thus truly universal, informed by existing approaches such as universal design. ${ }^{84} \mathrm{~A}$ non-judgemental, flexible and empathetic approach should be applied to all services for all socially excluded populations and beyond, in conjunction with a joined-up approach to health and social care. ${ }^{62} 7684$

The first three CMOCs (CMOC 1-3) were generated almost exclusively from grey literature sources and the other three (CMOC 4-6) were generated primarily from peer-reviewed literature. The grey literature included many high-level systems topics, including resourcing, funding cycles, the siloed nature of healthcare, health system goal setting and more, as well as describing patient-level factors. The majority of the peer-reviewed literature described patient-level healthcare interactions. In these interactions, contexts are often reflective of systematic or at least service-level planning, management, culture and leadership decisions and practices, but they were not explicitly examined. There is a need for more research exploring healthcare access for people who experience homelessness from a health systems perspective.

\section{Implications for policy and future research}

Based on the findings in the review, we recommend the following points for further exploration and research and future policy making in the area:

- Healthcare services should be funded using multiyear stable funding cycles (CMOC 2).

- Healthcare services for vulnerable populations should be the specific responsibility of a health system entity so it is not allowed to fall between different sectors or budget lines (CMOC 2 and CMOC 4).

- Healthcare for individuals experiencing homelessness should be provided in settings that do not allow stigma to dominate the culture. Trauma-informed practice and a deep understanding of the life experiences of socially excluded populations at all levels of an organisation or system are key, and these should be championed by leadership and management to create a culture that is accessible to all (CMOC 1 and CMOC 5).

- Healthcare should be easy to navigate for patients. Pathways, procedures and communication should all be highly coordinated and designed with patients' needs in mind. One-to-one support from key workers and peer advocates increases navigability (CMOC 4).

- Courses of treatment should be planned transparently in collaboration with the patient based on his or her full set of needs and wishes as a full person (CMOC 4).

- Healthcare should be provided in flexible settings with flexible rules to allow for meeting patients' life circumstances and needs where they are (CMOC 4 and CMOC 6).
- Healthcare system goals should be set with flexibility and complexity in mind (CMOC 1, CMOC 3 and CMOC 6).

- Providers and staff should be recruited based on their motivation in working with the patient population, and adequate training should be given to ensure that all staff and providers understand the needs of the population group (CMOC 1 and CMOC 6).

These recommendations will likely benefit most patients, housed or not, and as such investing in them would pay dividends for populations beyond those experiencing homelessness. However, for homeless populations these are crucial to ensuring that an already vulnerable group does not face further social exclusion when accessing healthcare.

\section{CONCLUSION}

Access to healthcare for populations experiencing homelessness depends on adequately resourcing and training providers to meet the particular needs of patients in a welcoming and attentive setting without stigma and judgement. Services should be closely linked, and staff and providers should be empowered to take responsibility for providing flexible, responsive and opportunistic care in flexible settings. For patients, having a good experience and getting one's needs met sets a precedent for future appropriate healthcare access. The contexts in which this is possible arise in a respectful, empathetic culture which is created when managers and leaders value and champion it.

Twitter Rikke Siersbaek @rikkesiersbaek, John Alexander Ford @JohnFord1849, Sara Burke @sburx and Clíona Ní Cheallaigh @clionani

Acknowledgements We would like to thank the members of the expert panel who provided feedback on the programme theory development and the conclusions of the review which strengthened and refined the study as a whole: Mike Allen, Marieke Altena, Dr Sara Burke, Dr Briege Casey, Dr Clíona Ní Cheallaigh, Dr Patrick O'Donnell and Niamh O'Rourke. We would also like to thank the reviewers for their helpful suggestions and comments which strengthened the article in many ways.

Contributors RS, CNC, SB, JAF and ST developed the research project. RS, JAF and CNC developed the search strategy with the help of a subject librarian. RS and JAF shaped and refined formal search strategies. RS carried out screening and article selection process, applied a realist logic of analysis to the data, built CMOCs and programme theories, presented to the expert panel and drafted the article. SB screened a subset of articles and facilitated the expert panel meeting. CNC added to the refinement of CMOCs and programme theory. JAF provided guidance and oversight of the realist analysis and added to the refinement of CMOCs and programme theory. CNC, SB, JAF and ST provided subject matter expertise and critical revisions at multiple stages. All authors read, reviewed and approved the final manuscript.

Funding This research was funded by the Health Research Board (SPHeRE/2013/1).

Disclaimer The views and opinions expressed herein are those of the authors and do not necessarily reflect those of the Health Research Board, Trinity College Dublin or the University of Cambridge.

Competing interests None declared.

Patient consent for publication Not required.

Provenance and peer review Not commissioned; externally peer reviewed.

Data availability statement All data relevant to the study are included in the article or uploaded as supplementary information. 
Supplemental material This content has been supplied by the author(s). It has not been vetted by BMJ Publishing Group Limited (BMJ) and may not have been peer-reviewed. Any opinions or recommendations discussed are solely those of the author(s) and are not endorsed by BMJ. BMJ disclaims all liability and responsibility arising from any reliance placed on the content. Where the content includes any translated material, BMJ does not warrant the accuracy and reliability of the translations (including but not limited to local regulations, clinical guidelines, terminology, drug names and drug dosages), and is not responsible for any error and/or omissions arising from translation and adaptation or otherwise.

Open access This is an open access article distributed in accordance with the Creative Commons Attribution Non Commercial (CC BY-NC 4.0) license, which permits others to distribute, remix, adapt, build upon this work non-commercially, and license their derivative works on different terms, provided the original work is properly cited, appropriate credit is given, any changes made indicated, and the use is non-commercial. See: http://creativecommons.org/licenses/by-nc/4.0/.

\section{ORCID iDs}

Rikke Siersbaek http://orcid.org/0000-0003-3223-1420

John Alexander Ford http://orcid.org/0000-0001-8033-7081

Sara Burke http://orcid.org/0000-0001-9419-1642

Clíona Ní Cheallaigh http://orcid.org/0000-0002-0842-425X

Steve Thomas http://orcid.org/0000-0001-9306-0114

\section{REFERENCES}

1 FEANTSA. What is ETHOS ? European Typology of Homelessness 2017;13.

2 'Reilly F O, Barror S, Hannigan A. Homelessness: an unhealthy state. health status, risk behaviours and service utilisation among homeless people in two Irish cities. Partnership for health equity 2015;97.

3 Argintaru N, Chambers C, Gogosis E, et al. A cross-sectional observational study of unmet health needs among homeless and vulnerably housed adults in three Canadian cities. BMC Public Health 2013;13:577.

4 Khandor E, Mason K, Chambers C. Access to primary health care among homeless adults in Toronto, Canada: results from the street health survey. Open Med 2011;5:94-103.

5 Fazel S, Geddes JR, Kushel M. The health of homeless people in high-income countries: descriptive epidemiology, health consequences, and clinical and policy recommendations. The Lancet 2014;384:1529-40.

6 Ní Cheallaigh C, Cullivan S, Sears J, et al. Usage of unscheduled hospital care by homeless individuals in Dublin, ireland: a crosssectional study. BMJ Open 2017;7:e016420.

7 O'Toole TP, Buckel L, Bourgault C, et al. Applying the chronic care model to homeless veterans: effect of a population approach to primary care on utilization and clinical outcomes. Am J Public Health 2010;100:2493-9.

8 Levesque J-F, Harris MF, Russell G. Patient-Centred access to health care: conceptualising access at the interface of health systems and populations. Int J Equity Health 2013;12:18.

9 Dixon-Woods M, Cavers D, Agarwal S, et al. Conducting a critical interpretive synthesis of the literature on access to healthcare by vulnerable groups. BMC Med Res Methodol 2006;6:35.

10 Davidson PL, Andersen RM, Wyn R, et al. A framework for evaluating safety-net and other community-level factors on access for lowincome populations. Inquiry 2004;41:21-38.

11 Evans DB, Hsu J, Boerma T. Universal health coverage and universal access. Bull World Health Organ 2013;91:546-546A.

12 Aday LA, Andersen R. A framework for the study of access to medical care. Health Serv Res 1974;9:208-20.

13 WHO. Everybody's business: strengthening health systems to improve health outcomes: WHO's framework for action. Geneva: WHO, 2007.

14 Cornes M, Whiteford M, Manthorpe J. Improving hospital discharge arrangements for people who are homeless: a realist synthesis of the intermediate care literature. Health Soc Care Community 2017:345-59.

15 Carver H, Ring N, Miler J, et al. What constitutes effective problematic substance use treatment from the perspective of people who are homeless? A systematic review and meta-ethnography. Harm Reduct J 2020;17.

16 Hwang SW, Ueng JJM, Chiu S, et al. Universal health insurance and health care access for homeless persons. Am J Public Health 2010;100:1454-61.

17 O'Carroll A, Wainwright D. Making sense of street chaos: An ethnographic exploration of homeless people's health service utilization. Int J Equity Health 2019;18:1-22.
18 O'Donnell P, Tierney E, O'Carroll A. Exploring levers and barriers to accessing primary care for marginalised GR discovery service for Trinity College Dublin (university). Int J Equity Health 2016;15:1-6.

19 Guarino K, Bassuk E. Working with families experiencing homelessness: understanding trauma and its impact. Zero to Three 2010;11:14-23.

20 Kertesz SG, McNeil W, Cash JJ, et al. Unmet need for medical care and safety net accessibility among Birmingham's homeless. $J$ Urban Health 2014;91:33-45.

21 Campbell DJT, O'Neill BG, Gibson K, et al. Primary healthcare needs and barriers to care among Calgary's homeless populations. BMC Fam Pract 2015;16:1-10.

22 Baggett TP, O'Connell JJ, Singer DE, et al. The unmet health care needs of homeless adults: a national study. Am J Public Health 2010;100:1326-33.

23 Salem B, Nyamathi A, Faith I. At a crossroads: reentry challenges and healthcare needs among homeless female Ex-Offenders. $J$ Forensic Nurs 2013;9:14-22.

24 Booth M, Bernard D, Quine S, et al. Access to health care among Australian adolescents young people's perspectives and their sociodemographic distribution. Journal of Adolescent Health 2004;34:97-103.

25 Harris M, Rhodes T. Hepatitis C treatment access and uptake for people who inject drugs: a review mapping the role of social factors. Harm Reduct J 2013;10:7.

26 Aidala AA, Wilson MG, Shubert V, et al. Housing status, medical care, and health outcomes among people living with HIV/AIDS: a systematic review. Am J Public Health 2016;106:e1-23.

$27 \mathrm{WHO}$. WHO / health systems strengthening glossary, 2020. Available: https://www.who.int/healthsystems/hss_glossary/en/index5.html

28 Pawson R. Evidence-based policy: a realist perspective. London: Sage Publications Inc, 2006.

29 Pawson R, Tilley N. Realistic evaluation. London: SAGE publications Inc, 1997.

30 Pawson R, Manzano-Santaella A. A realist diagnostic workshop. Evaluation 2012;18:176-91.

31 Wong G, Greenhalgh T, Westhorp G. RAMESES publication standards: realist syntheses. BMC medicine 2013;11:1-4.

32 Crisis. Critical condition: vulnerable single homeless people and access to GPs. London, UK: Crisis UK, 2002.

33 Davis J, Lovegrove M. Inclusion health: education and training for health professionals. London: Department of Health, 2016. https:// assets.publishing.service.gov.uk/government/uploads/system/ uploads/attachment_data/file/490870/NIHB_-_Inclusion_Health_ education_Report.pdf

34 Osborne B. Irish general practice: working with deprivation. Dublin: ICGP, 2015.

35 The Queen's Nursing Institute. Nursing care for people experiencing homelessness. London: The Queen's Nursing Institute, 2018.

36 Mungo's S. Health and homelessness: understanding the costs and role of primary care services for homeless people. London 2013.

37 . Inclusion health: improving the way we meet the primary health care needs of the socially excluded. London Social Exclusion Task Force, Cabinet Office; 2010. https://webarchive.nationalarchives. gov.uk/+/http:/www.cabinetoffice.gov.uk/media/346571/inclusionhealth.pdf

38 Page A, Hilbery O. Turning the Tide: A Vision Paper for multiple needs and exclusions. Adv Dual Diagn 2011;4:173-9.

39 Hudson BF, Flemming K, Shulman C, et al. Challenges to access and provision of palliative care for people who are homeless: a systematic review of qualitative research. BMC Palliat Care 2016;15:96

40 White BM, Newman SD. Access to primary care services among the homeless: a synthesis of the literature using the equity of access to medical care framework. J Prim Care Community Health 2015;6:77-87.

41 Gill P, Macleod U, Lester H. Improving access to health care for gypsies and travellers, homeless people and sex workers: An evidence-based commissioning guide for clinical commissioning groups and health \& wellbeing boards. London: Royal College of General Practitioners, 2013.

42 Wilson B, Astley P. Gatekeepers: access to primary care for those with multiple needs. Stoke-on-Trent: VOICES, Healthwatch and Expert Citizens, 2016.

43 The King's Fund. Delivering health and care for people who sleep rough: going above and beyond. London: The King's Fund, 2020.

44 Dawson A, Jackson D. The primary health care service experiences and needs of homeless youth: a narrative synthesis of current evidence. Contemp Nurse 2013;44:62-75.

45 Agency for Clinical Innovation. Consumer enablement guide: culturally responsive practice, 2020. Available: https://aci.health.nsw. 
gov.au/resources/primary-health/consumer-enablement/guide/howto-support-enablement/culturally-responsive-practice

46 Faculty for Homeless and Inclusion Health. Homeless and inclusion health standards for commissioners and service providers. London: Pathway, 2018. https://www.pathway.org.uk/wp-content/uploads/ Version-3.1-Standards-2018-Final.pdf

47 Mahon E. More than a caring personality: factors affecting staff retention in non profit organisations in Ireland. Ir Soc Work 2016;Spring:45-50 https://www.lenus.ie/bitstream/handle/10147/ $617869 /$ MoreThan. pdf? sequence $=1$ \&isAllowed $=y$

48 Cortis N, Blaxland M. Workforce issues in specialist homelessness services. Sydney: Social Policy Research Centre, UNSW Sydney, 2017.

49 Focus Ireland. Recommendations to government: budget 2020. DublinFocus Ireland; 2019. https://www.focusireland.ie/wp-content/ uploads/2019/09/Budget-2020-Policy-Document.pdf

50 Making Every Adult Matter Coalition. Solutions from the frontline. London MEAM Coalition; 2015. http://meam.org.uk/wp-content/ uploads/2013/04/Solutions-from-the-Frontline-WEB.pdf

51 Homeless Link, St Mungo's. Improving hospital admission and discharges for people who are homeless. London Homeless Link \& St Mungo's; 2012. https://www.homeless.org.uk/sites/default/ files/site-attachments/HOSPITAL_ADMISSION_AND_DISCHARGE. REPORTdoc.pdf

52 Klop HT, de Veer AJE, van Dongen SI, et al. Palliative care for homeless people: a systematic review of the concerns, care needs and preferences, and the barriers and facilitators for providing palliative care. BMC Palliat Care 2018;17:67.

53 Robards F, Kang M, Usherwood T, et al. How Marginalized young people access, engage with, and navigate health-care systems in the digital age: systematic review. J Adolesc Health 2018;62:365-81.

$54 \mathrm{Lamb} \mathrm{J}$, Bower P, Rogers A, et al. Access to mental health in primary care: A qualitative meta-synthesis of evidence from the experience of people from "hard to reach" groups. Health 2012;16:76-104.

55 Sumalinog R, Harrington K, Dosani N, et al. Advance care planning, palliative care, and end-of-life care interventions for homeless people: a systematic review. Palliat Med 2017;31:109-19.

56 Weber J, Lee RC, Martsolf D. Understanding the health of Veterans who are homeless: a review of the literature. Public Health Nurs 2017;34:505-11.

57 Crock E. Access to healthcare services for people living with HIV experiencing homelessness - a literature review. Aust J Adv Nurs 2016;34:42-51.

58 Leonard M. Homeless healthcare in England - how the experience of homelessness makes a difference. Homeless in Europe 2019;Winter:7-8 https://www.feantsa.org/public/user/Resources/ magazine/2019/Winter/Homeless_in_Europe_-_Article_3-_Homeless healthcare_in_England_\%E2\%80\%93_how_the_experience_of_ homelessness_makes_a_difference_-_Mark_leonard.pdf

59 Elliott AS, Adolescent Health Committee. Meeting the health care needs of street-involved youth. Paediatr Child Health 2013;18:317-21.

60 Hamilton K, Tolfree R, Mytton J. A systematic review of active casefinding strategies for tuberculosis in homeless populations. int $j$ tuberc lung dis 2018;22:1135-44.

61 Brown A, Rice SM, Rickwood DJ, et al. Systematic review of barriers and facilitators to accessing and engaging with mental health care among at-risk young people. Asia-Pacific Psychiatry 2016;8:3-22.

62 Luchenski S, Maguire N, Aldridge RW, et al. What works in inclusion health: overview of effective interventions for marginalised and excluded populations. The Lancet 2018;391:266-80.

63 Wallace C, Farmer J, McCosker A. Community boundary spanners as an addition to the health workforce to reach marginalised people: a scoping review of the literature. Hum Resour Health 2018;16:46.

64 O'Donnell P, Tierney E, O'Carroll A. Exploring levers and barriers to accessing primary care for marginalised groups and identifying their priorities for primary care provision: a participatory learning and action research study. Int J Equity Health 2016;15:1-16.

65 White BM, Logan A, Magwood GS. Access to diabetes care for populations experiencing homelessness: an integrated review. Curr Diab Rep 2016;16:112.

66 O'Donnell P, Tierney E, O'Carroll A, et al. Exploring levers and barriers to accessing primary care for marginalised groups and identifying their priorities for primary care provision: a participatory learning and action research study. Int $J$ Equity Health 2016;15:197.

67 Rural and Regional Health and Aged Care Services. Cultural responsiveness framework: guidelines for Victorian health services. Melbourne, 2009. Available: http://www.health.vic.gov.au/cald

68 Seeleman C, Essink-Bot ML, Stronks K. How should health service organizations respond to diversity? A content analysis of six approaches health policy, reform, governance and law. BMC Health Serv Res 2015;15:1-8.

69 Rural and Regional Health and Aged Care Services. Cultural responsiveness framework: guidelines for Victorian health services. Melbourne: Rural and Regional Health and Aged Care Services, 2009.

70 McMillan F. Position paper: culturally responsive health care. ACT: Indigenous Allied Health Australia, 2013.

71 Jones C, Perera A, Chow M, et al. Cardiovascular disease risk among the poor and homeless - what we know so far. Curr Cardiol Rev 2009;5:69-77.

72 John W, Law K. Addressing the health needs of the homeless. Br J Community Nurs 2011;16:134-9.

73 Kaduszkiewicz $\mathrm{H}$, Bochon $\mathrm{B}$, van den Bussche $\mathrm{H}$. The medical treatment of homeless people. Dtsch Arzteblatt Int 2017;114:673-9.

74 Homeless Link. The unhealthy state of homelessness. London: Homeless Link, 2014.

75 Islam MM, Conigrave KM. Assessing the role of syringe dispensing machines and mobile van outlets in reaching hard-to-reach and highrisk groups of injecting drug users (IDUs): a review. Harm Reduct $J$ 2007;4:14-19.

76 Martens WHJ. Vulnerable categories of homeless patients in Western societies: experience serious barriers to health care access. Med Law 2009;28:221-39.

77 Wong G, Greenhalgh T, Westhorp G, et al. Development of methodological guidance, publication standards and training materials for realist and meta-narrative reviews: the RAMESES (realist and Meta-narrative evidence syntheses - evolving standards) project. Health Serv Deliv Res 2014;2:1-252.

78 Tankimovich M. Barriers to and interventions for improved tuberculosis detection and treatment among homeless and immigrant populations: a literature review. J Community Health Nurs 2013;30:83-95.

79 Lutge EE, Wiysonge CS, Knight SE, et al. Incentives and enablers to improve adherence in tuberculosis. Cochrane Database Syst Rev 2015;110.

80 Pawson R. Digging for nuggets: How "bad" research can yield "good" evidence. Int J Soc Res Methodol 2006;9:127-42.

81 Ford JA, Wong G, Jones AP. Access to primary care for socioeconomically disadvantaged older people in rural areas: a realist review. BJM Open 2018;13:e0193952.

82 Levesque J-F, Harris MF, Russell G. Patient-centred access to health care: conceptualising access at the interface of health systems and populations. Int J Equity Health 2013;12:1.

83 Bramley EG, Fitzpatrick S, Edwards J. Hard edges mapping severe and multiple disadvantage. London: LankellyChase Foundation, 2015. www.lankellychase.org.uk

84 HSE and the National Disability Authority. National guidelines on accessible health and social care services. Dublin: HSE and the National Disability Authority, 2014. 